\title{
Real-time separation of multineuron recordings with a DSP32C signal processor
}

\author{
Rainer Gädicke *, Klaus Albus \\ Department of Neurobiology, Max Planck Institute for Biophysical Chemistry, D-37077 Göttingen, Germam \\ Reccived 27 April 1994; revised 8 August 1994; accepted 19 August 1994
}

\begin{abstract}
We have developed a hardware and software package for real-time discrimination of multiple-unit activities recorded simultaneously from multiple microelectrodes using a VME-Bus system. Compared with other systems cited in literature or commercially available, our system has the following advantages. (1) Each electrode is served by its own preprocessor (DSP32C); (2) On-line spike discrimination is performed independently for each electrode. (3) The VME-bus allows processing of data received from 16 electrodes. The digitized $(62.5 \mathrm{kHz})$ spike form is itself used as the model spike; the algorithm allows for comparing and sorting complete wave forms in real time into 8 different models per electrode.
\end{abstract}

Keywords: Multi-unit recording; DSP32C signal processor; Computer separation of waveforms; Complete spike form matching

\section{Introduction}

A low-impedance microelectrode positioned in the proximity of active neurons will record several distinct waveforms, each of which can be assigned to an individual neuron. This is called multi-unit or multineuron recording. A prereqisite for analyzing the activity pattern of each individual neuron contained in this recording, and for investigating possible interactions between these neurons, is to separate the multi-unit recording into single-unit activity. A large number of hardware and software solutions for solving the sorting problem have been developed in the past, among them amplitude separation, Fourier analysis, and template matching (for review see Schmidt, 1984a,b). Recent developments have applied analog correlation technique (Wörgötter et al., 1986) or reduced template matching (Kindermann et al., 1987; Salganicoff et al., 1988; Sarna et al., 1988; Kreiter et al., 1989).

Digital signal processors at moderate prices have been available for several years. The first signal processor designed for floating point calculations was the DSP32C. We have utilized the high processing speed

\footnotetext{
* Corresponding author. Tel.: 551-201-652; Fax: 551-201-788.
}

of the DSP32C to implement a robust algorithm for waveform discrimination. The digitized spike form itself is used as the model spike, and new spikes are assigned to a particular class when they match the respective model spike. Each electrode is served by its own preprocessor and the algorithm allows for comparing and sorting complete waveforms in real time into 8 different classes per electrode. The results have been published in abstract form (Gädicke and Albus, 1993).

\section{Principle of operation}

Extracellular recordings with electrodes positioned close $(<100 \mu \mathrm{m})$ to neuronal somata teveal 2 types of waveforms (Humphrey, 1979): negative-positive spikes (Fig. 1c), and positive-negative spikes (Fig. 1d). The latter waveform is recorded close to the apical dendrite of pyramidal cells, whereas the former waveform is recorded around stellate cells and around pyramidal cells, either at points superficial to the apical dendrite or below the soma. High-pass filtering $(>160 \mathrm{~Hz})$ as performed in our approach (see below, implementation) changes waveforms by adding an additional half wave and by pronouncing inflections at the rising phase of the initial component of the spike (Figs. 1f,g). Such inflections are thought to represent the transition from 


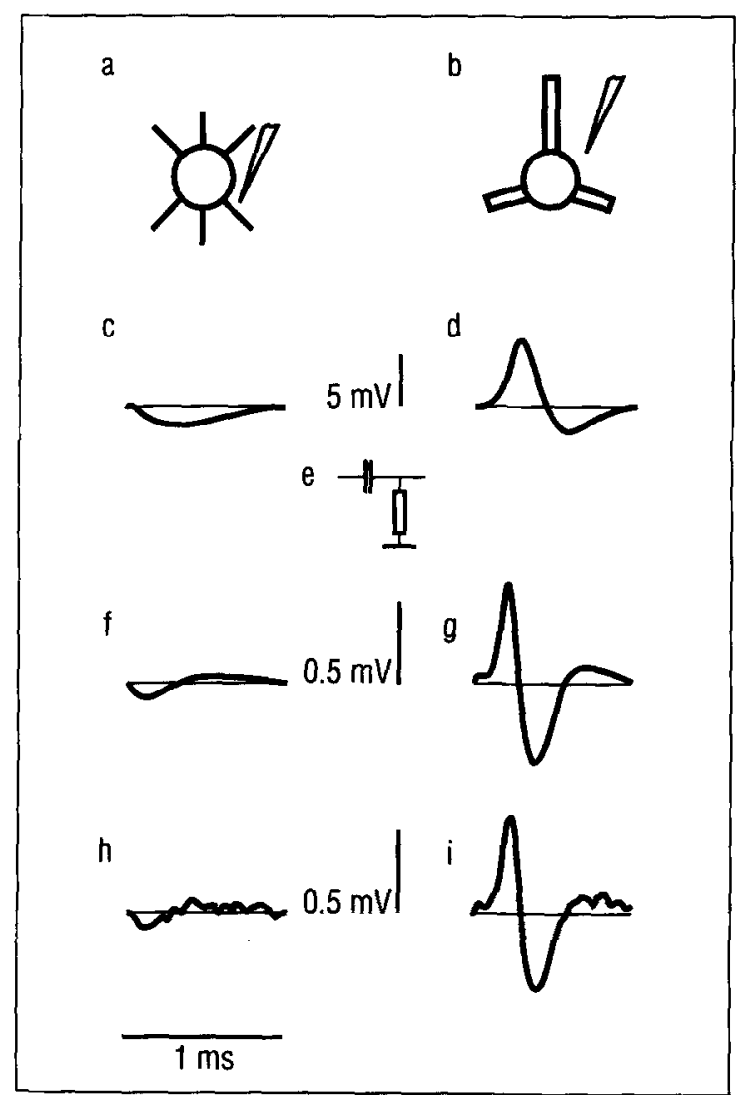

Fig. 1. Effects of high-pass filtering on the shape of action potentials. a: stellate cell. b: pyramidal cell. c,d: unfiltered action potentials. e: high-pass filter 4th order $(>160 \mathrm{~Hz})$. f,g: high-pass filtered action potentials. h,i: high-pass filtered action potentials with noise added.

the axon hillock to the somatic spike and are seen also in recordings from presumably stellate neurons (Humphrey, 1979).

The high-pass filtered signal is digitized at $62.5 \mathrm{kHz}$. The sorting process consists of 3 phases: (1) determination of DC voltage offset and spike thresholds, (2) definition of model spikes, and (3) real time sorting.

\subsection{Determination of $D C$ voltage offset and spike thresh- olds}

A 1-s period of the recording is used to calculate a DC offset voltage. The mean value of the 62,500 data points is subtracted from each subsequent data point, thus compensating for possible DC shifts of the differential amplifier and the AD board. The offset value is redetermined whenever a new real-time sorting run (phase 3) is started. Next, a mean noise level is calculated from $62,372(62,500-128)$ single values by

$\mathrm{MNL}=\frac{1}{N-W} \sum_{n=1}^{N-W}\left|x_{n+\frac{W}{2}}-\left(\frac{1}{W} \sum_{m=1}^{W} x_{n+m}\right)\right|$

where $\mathrm{MNL}=$ mean noise level, $N=$ total number of

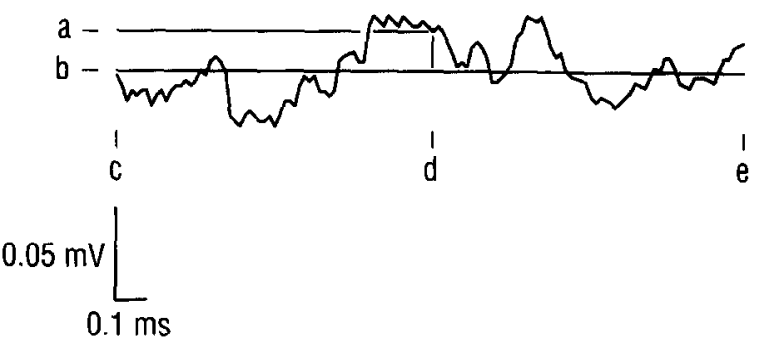

Fig. 2. Calculation of mean noise level (MNL), a: amplitude of noise at 65 th data point $(\mathrm{d})$. b: instantaneous $\mathrm{DC}$ voltage calculated from 128 data points or 2048 us (c-e).

samples $=62,500, W=$ number of samples in a window $=128$, and $x_{i}=$ sample $i$.

Thus, using a sliding window of 128 data points, these values are calculated by subtracting the mean of the window from the value at the middle of the window (Fig. 2). The sliding window technique is used in order to attenuate frequencies preferentially greater than 500 $\mathrm{Hz}$. The MNL is calculated from the sum of the absolute values of each of the 62,372 values. The MNL is then multiplied by constants to calculate thresholds for waveform detection. The constants were determined empirically and are as follows (Fig. 3):

threshold for 'start spike sampling' $\quad=8 \times \mathrm{MNL}$; threshold for 'enable new spike sampling' $=6 \times \mathrm{MNL}$; 'shifted zero line' $=4 \times \mathrm{MNL}$.

These thresholds are set initially by the computer and may then be manually adjusted by the experimenter. Short time periods of the ongoing recording

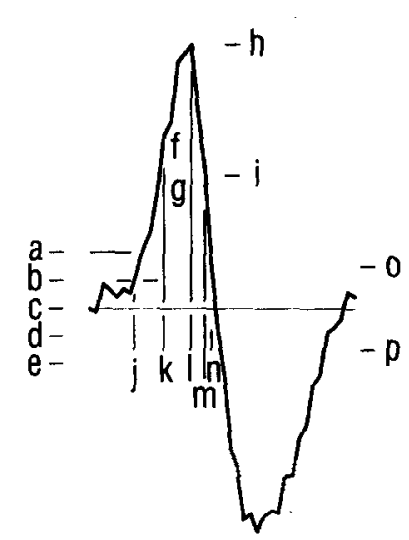

Fig. 3. Thresholds for spike detection and waveform parameters used for defining model spikes. a: positive 'start spike sampling' threshold. b: positive 'shifted zero line'. c: zero voltage. d: negative 'shifted zero line'. e: negative 'start spike sampling' threshold. The following parameters are indicated only for the first component of the spike. $f$, area; g, squared area $(+$, for positive going spike components; for negative going spike components); $h$, maximal amplitude; $i$, half maximum amplitude; $j$, shifted zero crossing +1 data point $=$ start of spike; $k$, time at half maximum amplitude rising phase; 1 , time at maximum amplitude; $m$, time at half maximum falling phase; $n$, end of first component; $o$, positive 'enable new spike sampling' threshold; p, negative 'enable spike sampling' threshold. 


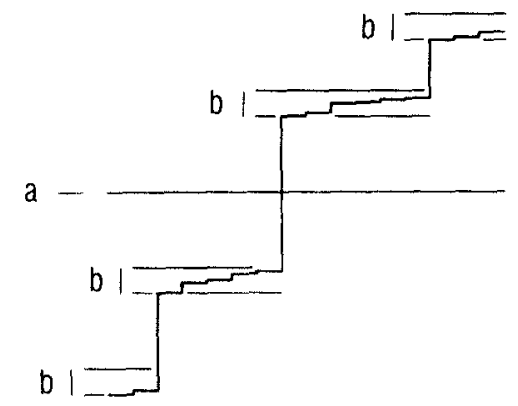

Fig. 4. Arrangement of 1 of the parameters shown in Fig. 3 according to size. A total of 16 values is shown. Values within ranges $b$ are selected to represent 1 model spike. Values below line a are negative, values above line a are positive.

containing spikes are displayed on a monitor, along with the thresholds. Only spikes with a maximum amplitude of at least $70 \%$ of the 'start spike sampling' threshold are displayed. The thresholds are used during phases 2 and 3 (below).

\subsection{Definition of model spikes}

The basis for defining model spikes are 259 segments (3-ms sampled time periods), each containing at least 1 complete spike. Sampling of 1 segment is started when the mean value of 2 consecutive data points is more positive than the positive threshold or more negative than the negative threshold for 'start spike sampling'. The sampled data for $1 \mathrm{~ms}$ prior to and $2 \mathrm{~ms}$ subsequent to the threshold crossing is stored by the host computer. After 1 segment has been sampled the 'enable new spike sampling' threshold becomes effective. The computer searches for incoming spikes only if 8 consecutive data points subsequent to a segment have been recorded which are either less positive than the positive threshold or less negative than the nega- tive threshold. Thus spikes starting in the last few 100 $\mu \mathrm{s}$ of a segment will not be accepted as starting the sampling of a new segment. When sampling of the 259 segments has been completed, the procedure of defining model spikes begins by selecting a time period of 2 ms out of each 3-ms segment saved in the host computer. The computer starts at the crossing point of the 'start spike sampling' threshold and searches back in time towards the first crossing of the 'shifted zero line' (shifted zero crossing). The time of the data point following the shifted zero crossing is defined as the actual starting time of the spike and the following $2 \mathrm{~ms}$ are used for defining the model spikes. The 'shifted zero line' is not used to start spike sampling because it is occasionally crossed by higher amplitude noise or by small amplitude spikes having a signal-to-noise ratio too small to consider them for further discrimination. Implementation of the 'start spike sampling' threshold avoids inclusion of these small potentials into the sample used for defining model spikes and thus saves computing time. The 259 spikes (i.e., the 2-ms time periods containing the spikes) may be graphically displayed.

Each of the 259 spikes is separated into its components (half waves) above and below the zero line voltage. Components having an area of less than (16 MNL $\times 16$ data points) (unit area is Vs) are not accepted for further evaluation; a maximum of 3 components per spike can be analyzed. Each component is then characterized by 9 parameters (for details see Fig. 3) so that for each spike a maximum of 27 parameters may be available. The values of each parameter are presented in a graph as shown in Fig. 4. The $y$ axis of the graph is the magnitude of the parameter. By manually adjusting $a$ range at the ordinate ( $b$ in Fig. 4 ) values of a given parameter can be selected. The mean of the spikes from which these parameters were computed is defined
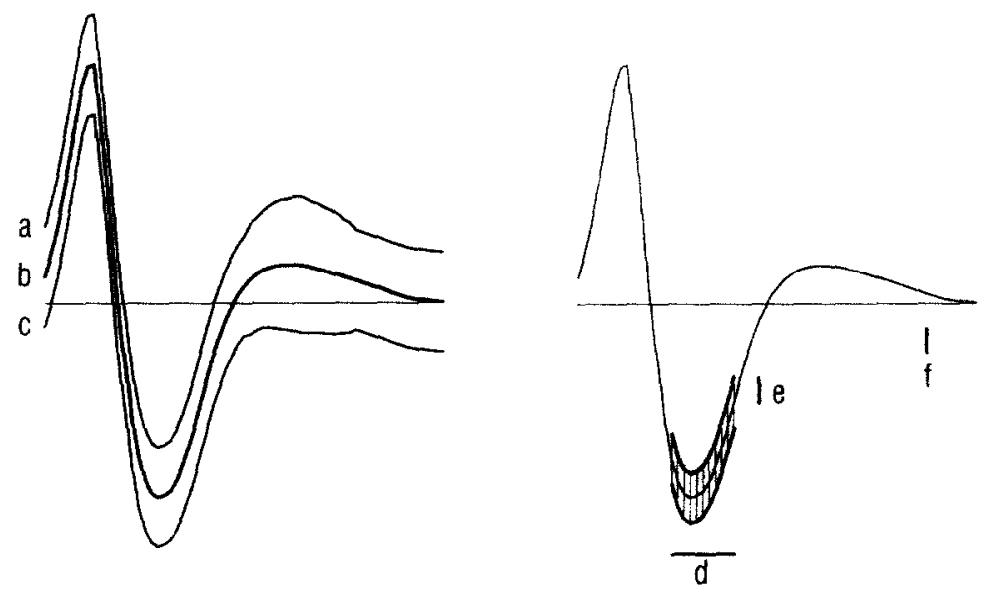

Fig. 5. (left): model spike defined on the basis of a parameter selection as shown in Fig. 4. a: mean value +1 SD. b: mean value. c: mean value $-1 \mathrm{SD}$. (right): further setting the model spike. d: matching period. e: confidence limit for matching incoming spikes. f: time mark for spike end. For further explanation see text. 
as a model spike. All parameters are displayed on the monitor. The experimenter selects the one with the most distinct staircase pattern. A staircase with a profile as in Fig. 4 indicates that on the basis of this particular parameter 4 different model spikes can be differentiated. The spikes are then grouped by parameter range, and the mean and standard deviation of the spikes are displayed for each group (Fig. 5, left). Each model spike thus represents both the average shape of a particular group of spikes and, in addition, the variability in the time course of the individual spikes of this group. For 1 electrode up to 8 different model spikes can be defined.

After the computer presents the estimated model spikes, the experimenter can alter them, for example, by uniting 2 different models into 1 or by further subdividing 1 model into several. The computer allows the experimenter to inspect individual spikes on the monitor and switch the $x$ axis between time and parameter value. The final decision on whether a waveform should be accepted as model is left to the experimenter. Waveforms not accepted as a model are ignored and their data points are not considered any further.

After the final shape of the models has been defined the experimenter must set several parameters before the actual sorting procedure is started. Usually the entire spike is used for the matching procedure (see Fig. 9). However, only a short period of a spike can be used for the matching procedure as well, if this spike clearly differs in shape from the other model spikes (Fig. 5, right). The experimenter can define the beginning and end of the matching period. The experimenter can also set confidence limits for matching of incoming spikes. These limits are normally set to the standard deviations of the model spikes. Finally a time mark must be set (spike end) which must be passed in order for the 'enable new spike sampling' threshold to become effective (see above). Setting of this time mark is necessary in cases where the matching period comprises only a short period of a spike.

\subsection{Real-time sorting phase}

The computer downloads all necessary parameters (model spikes and thresholds) to the preprocessor and the sorting procedure is started. The comparison of an incoming spike with the model spikes is performed in 2 steps. The first step synchronizes the incoming spike with spike models, the first component of which have the same polarity as the new spike (Fig. 6). This synchronization is necessary because the time course of a single spike, in particular when it starts diving out of the noise, may deviate to some extent from the model spike.

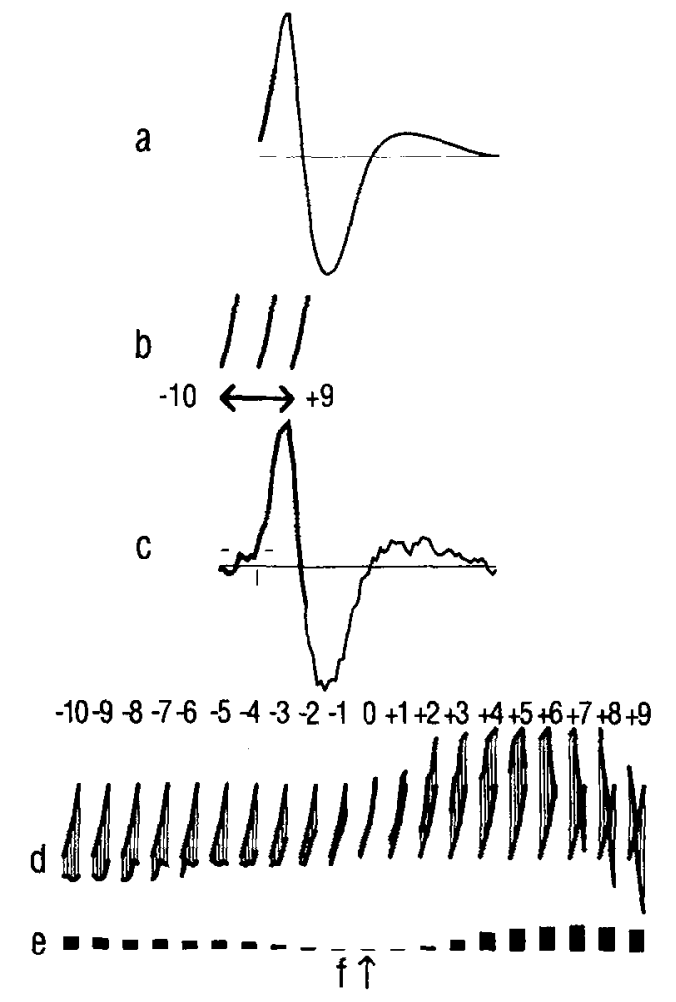

Fig. 6. Synchronization of incoming spike with model spike. a: model spike. The thicker part of the rising phase of the first component (b) is shifted in time relative to the incoming spike (c) backwards and forwards by starting at a point where the latter crosses the shifted zero line. d: graphical display of point differences between model spike and newly incoming spike. e: each filled rectangle represents the sum of the squared point differences for each of the 20 positions tested. The arrow in $f$ points to the smallest of these values. For further explanation see text.

Synchronization shifts the data up to 10 sample intervals forward or backwards from the time of occurance of the 'shifted zero crossing' by the new incoming spike. At each position 5 data points are used for making the comparison (Fig. 6d). The 5 point differences between the new spike and the model spike are squared and are summed up for each of the 20 points in time (Fig. 6e). The minimum of the 20 values is the point of best fit between new spike and model spike; the first of the 5 points of a minimum indicates the time of occurrence of the new spike and is stored in the preprocessor for each of the models tested.

The second step is the actual match/mismatch test (Fig. 7) which again is only performed with models having a first component of the same polarity as the new spike. The test is performed at 5 different positions (Fig. 7d), which are distributed around a point in time set by the synchronization procedure shown in Fig. 6. The number of point distances determined per position corresponds to the number of data points of the matching range. The point distances between model spike and new spike at each position are squared and summed. The smallest of the 5 values is compared with 


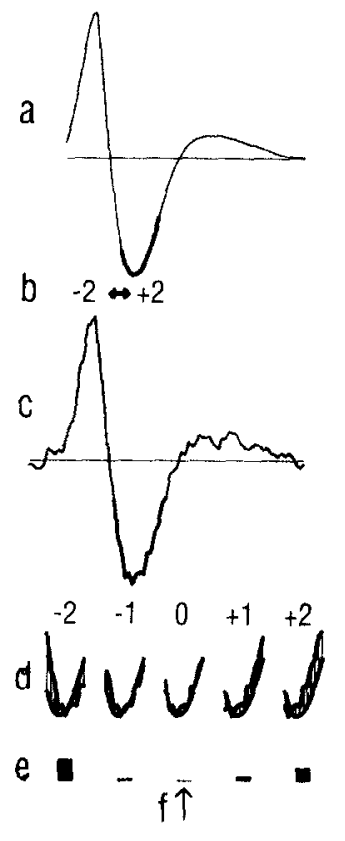

Fig. 7. Match/mismatch test. For further explanation see text and Fig. 6 .

the sum of the squared point distances and 1 confidence limit (matching range). The new spike is matched with that model from which it deviates least.

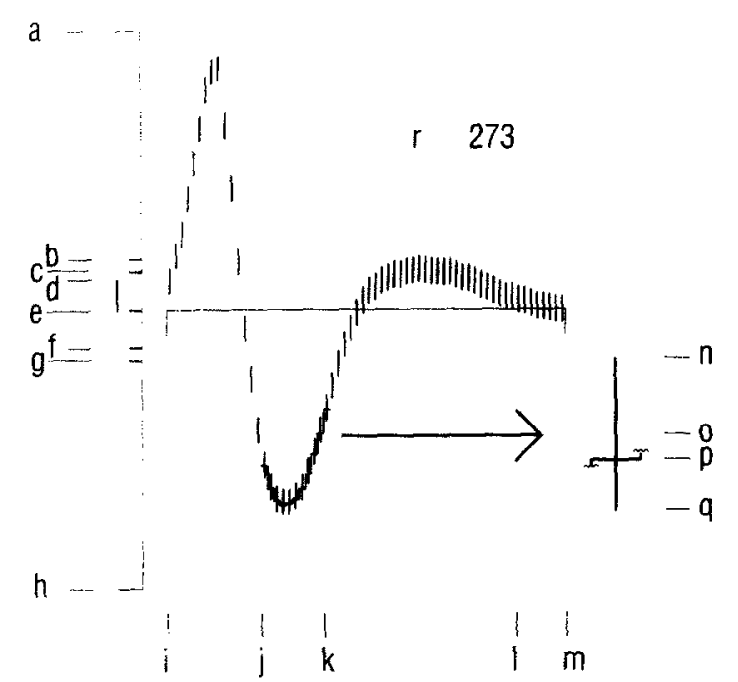

Fig. 8. Graphical display of the result of a real-time separation of multi-unit activity. a-h: maximal amplitude range set for all models recorded during 1 discrimination. b; positive 'start spike sampling' threshold. c: positive 'enable new spike sampling' threshold. d: confidence limit for matching of new spikes; corresponds to e in Fig. 5. e: zero voltage. f: negative 'enable new spike sampling' threshold. g: negative 'start spike sampling' threshold. $\mathrm{i}-\mathrm{m}$ : time axis (64 data points $=1024 \mathrm{us}$ ). $\mathrm{j}$ : start of matching period. $\mathrm{k}$ : end of matching period. 1: time mark for spike end; corresponds to $f$ in Fig. 5 . n: mean value $+1 \mathrm{SD}$ of the spikes matched with the model. 0 : mean value of spikes matched with the model. p: model spike. $q$ : mean value -1 SD of the spikes matched with the model. r: number of spikes matched with the model spike.
If the deviation of the new spike from the model spike exceeds the matching ranges of all model spikes the new spike is counted as an outlier. If matched with a particular model the time of occurrence of the new spike and the number of the model spike are transferred to the host computer. The preprocessor maintains a running average of spikes sorted into each class. During recording of multi-unit activity the experimenter thus can inspect the mean and standard deviation of each class of spikes.

The result of the sorting procedure is shown for 1 class of spikes in Fig. 8. The model spike and the mean and the standard deviation of the spikes matched to this model are graphically displayed. In addition the thresholds set in phase 3 are indicated. In Fig. 9 the result of a sorting procedure is demonstrated which was applied to simultaneous recordings from 3 tungsten microelectrodes in the striate cortex of an anesthetized and immobilized cat. The units were activated by stimulating the retina with patterned light. During a recording time of $24 \mathrm{~min}, 6633$ action potentials were recorded, 5529 of which were matched to a total of 14 different model spikes. 1104 spikes $(16.6 \%)$ did not fulfil the matching criteria and were counted as outliers (class zero). The unit matched to class 1 at electrode zero began changing its shape soon after starting the sorting procedure. Shortly after completion of the recording the unit was lost entirely.

\section{Implementation}

A 21-slot VME-Bus system is used. The signal from each microelectrode is amplified approximately 5000 times, galvanically isolated, and filtered using 4 th order low- and high-pass filters. Low-frequency signals (EEG, $<120 \mathrm{~Hz})$ are fed directly into the A/D board of the VME-Bus system (MPV 954) and are sampled at 244 $\mathrm{Hz}$. The phase shift due to filtering is minimal between 1 and $20 \mathrm{~Hz}$. The high-frequency signal passes through an analog Bessel filter with a corner frequency of 160 $\mathrm{Hz}$ and is sampled at $62.5 \mathrm{kHz}$ with a resolution of 12 bits. Sampled data values are buffered by a 2048-element FIFO memory before being read by the DSP32C preprocessor. Each preprocessor board contains 2 DSP32Cs, each with 8192 32-bit words of high-speed memory. The preprocessors are programmed in assembly language.

The preprocessor boards are contained in a 21-slot VME-Bus system controlled by a host computer running UNIX. The host computer is programmed in Modula-2 (Brown and Bauer, 1990). The host has direct memory access (DMA) to each preprocessor, which is used by the host to read information from an output ring buffer in the DSP32C memory.

During spike sorting each preprocessor passes 32 
bits of information to the host for each spike. The information contains the sample clock number, the model spike number, and some control information. If the DSP32C input FIFO overflows, the host is notified as well.

\section{Discussion}

Since the host computer reads messages directly from the memory of the DSP32C preprocessors, the preprocessors can spend all their time performing spike discrimination. They do not have to service the host computer. Each preprocessor can discriminate among 8 model spikes. The DSP32C is fast enough unless all 8 model spikes have the same polarity and spikes occur continuously at more than $300 \mathrm{~Hz}$. Higher spike rates could be accommodated using either a faster preprocessor or a lower sampling rate.

Digital filtering of low-frequency signals can be realized using a faster preprocessor. This would allow direct filtering of EEG without phase shift.
0

0

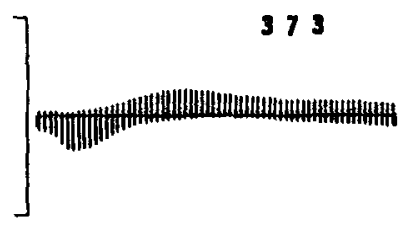

1

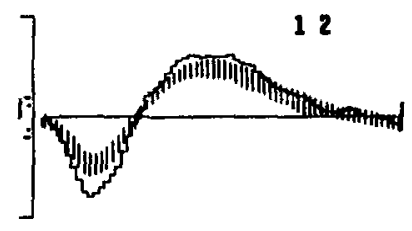

2

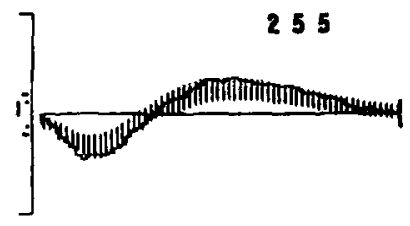

3

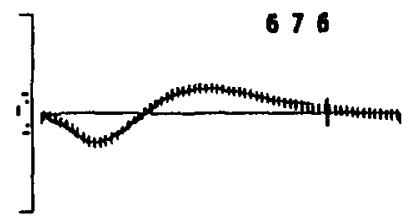

4

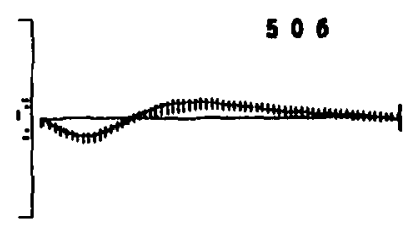

255
1
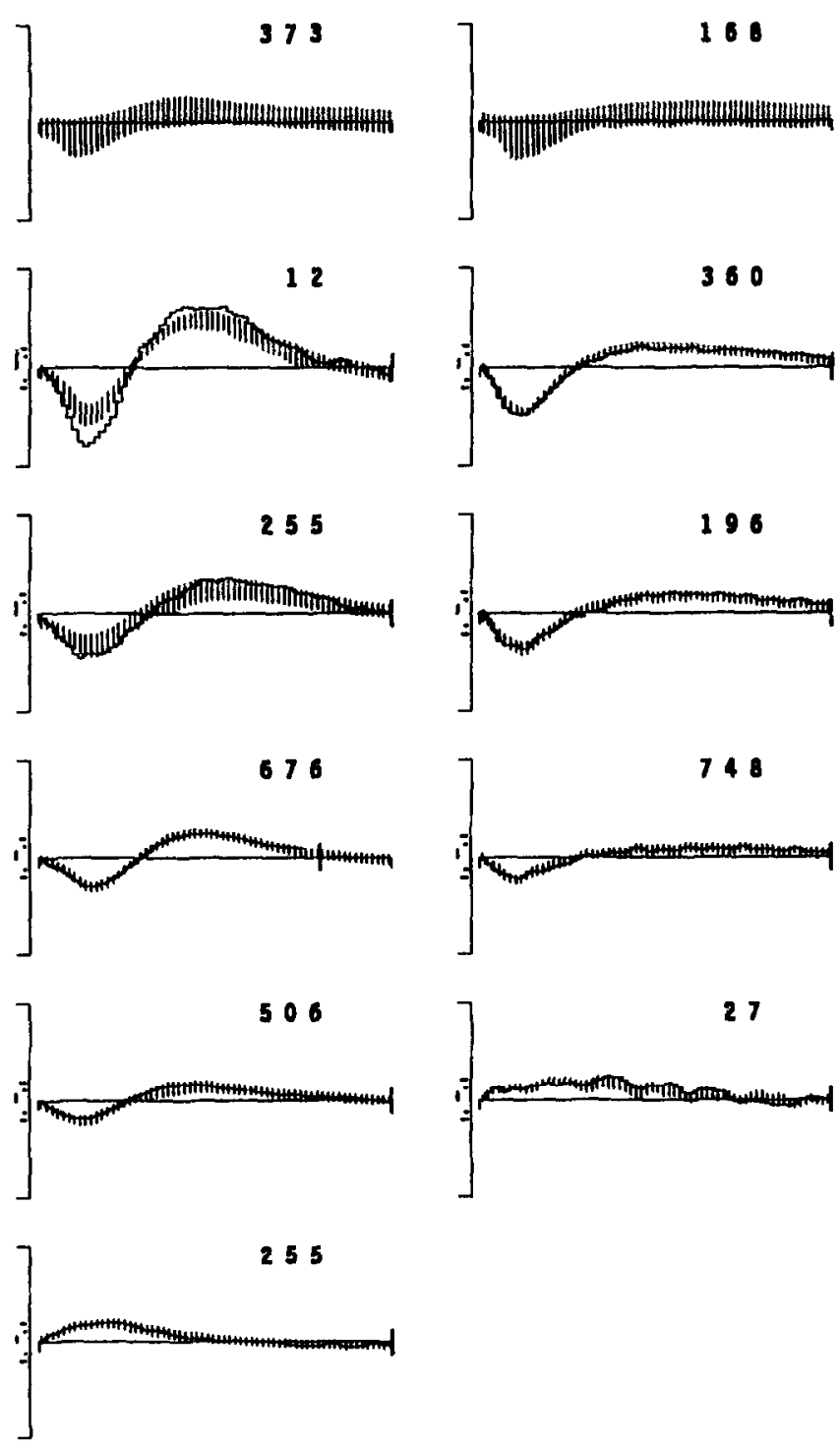

2
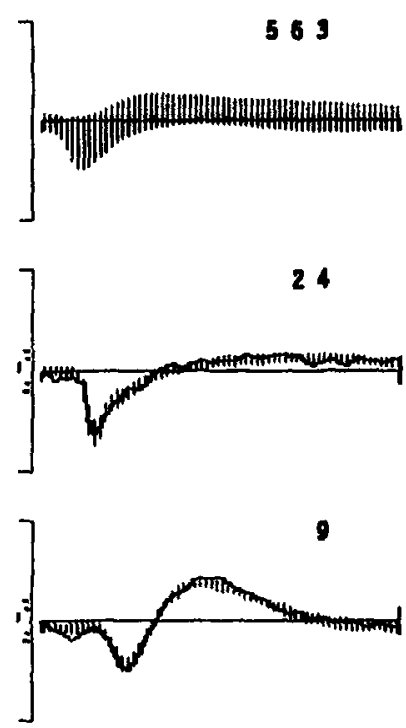

771

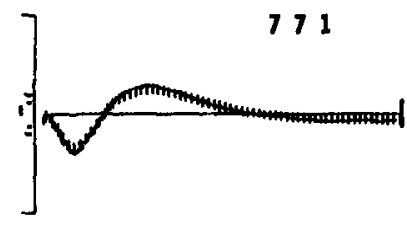

835

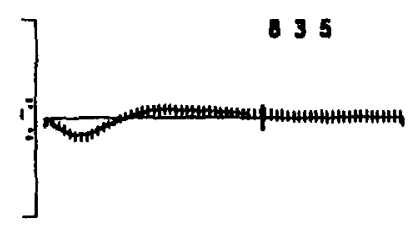

65

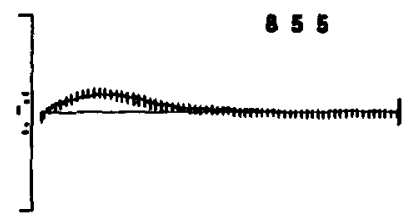

Fig. 9. Result of an in vivo experiment (anesthetized and immobilized cat, light stimulation). Multi-unit activity in the striate cortex was simultaneously recorded with 3 low-impedance tungsten microelectrodes. Model spikes (continuous lines) and \pm 1 SD of matched spikes (vertical bars) are arranged into 3 vertical columns 0,1 and 2 , and each of the columns represents the spikes recorded from 1 electrode. The different models (or classes of spikes) are denoted by numbers shown in the column on the left hand side. Number 0 denotes a class containing potentials not matched with any of the model spikes (outliers). For further explanations see Fig. 8 and text. Abszissa in each histogram is $1024 \mu \mathrm{s}$ long, the maximal range of the ordinate is $\pm 1 \mathrm{mV}$. 
The system has been tested so far quantitatively, by using artificial spikes without noise, and qualitatively, by comparing the results of the sorting procedure with storage oscilloscope recordings of single, large amplitude spikes bursting at high frequencies. In the latter case matching of new spikes depends on the matching range assigned to the model spike; however, strongly depolarized spikes with delayed inflections at the rising phase of their initial component usually are counted as outliers. With artificial spikes amplitude differences of less the $5 \%$ are reliably discriminated. The system has not been designed to detect spikes overlapping in time; if such events occur, the resulting waveform is counted as an outlier.

The proportion of outliers usually is less than $5 \%$ of the total number of spikes recorded. There are exceptions to this rule however. In the case shown in Fig. 10 about $16 \%$ of the units were counted as outliers. Since this proportion varied with the type of light stimulation, we assume that the respective units were present from the beginning but not among the 259 spikes collected during phase 2 to define models. Alternatively these units might have joined the set of multiple units only after phase 2 had been completed. An option could be added to the system to allow for off-line inspection and sorting of the outlier waveforms (see also Atiya, 1992). A false match (i.e., a spike belonging to one template is incorrectly assigned to another template) is unlikely to occur if the running average during the actual sorting procedure displays means within the confidence limits of the model spike. If a change occurs, the discrimination procedure can be stopped and the data not considered for further evaluation.

The system in its present configuration gives the experimenter a good chance to visually control and adjust thresholds and model spike parameters. Once the 259 samples have been collected, the time needed to define model spikes depends somewhat on the number of models present, but is on average $1 \mathrm{~min}$ per electrode. There is still room for automating procedures currently performed by the experimenter. However, working with 3 or more microelectrodes simultaneously (the system in its present configuration would accept 16 electrodes) is conditional not only on more automation, but more urgently on development of methods to suitably display and evaluate the data mass.

\section{Acknowledgements}

We thank Dan Brown for his critical reading of, and helpful comments on, the manuscript.

\section{References}

Atiya. A.F. (1992) Recognition of multiunit neural signals, IEEE Trans. BME, 39: 723-729.

Brown, D. and Bauer, K. (1990) PowerMod - System Guide, Internal Paper, Max-Planck-Institut für Medizinische Forschung, Hcidelberg.

Gädicke, R. and Albus, K. (1993) Real time discrimination of multiple unit activities recorded simultaneously from multiple microelectrodes using a VME-Bus system. World Congress on Neural Networks, Portland, OR, Vol. 4, pp. 729-731.

Humphrey, D.R. (1979) Extracellular, single-unit recording methods. In: Electrophysiological Techniques, Short Course, Society for Neuroscience, Atlanta, GA, pp. 199-159.

Kindermann, L., Redies, H. and Sieben, U. (1987) Real time analysis of the activity of small neuronal networks with one multi-unit electrode. In: N. Elsner and O.D. Creutzfeldt (Eds.), New Frontiers in Brain Research, Proc. 15th Göttingen Neurobiology Conference, Thieme, Stuttgart, p. 106.

Kreiter, A.K., Aertsen, A.M.H.J. and Gerstein, G.L. (1989) A lowcost single board solution for real-time, unsupervised waveform classification of multineuron recordings, J. Neurosci. Methods, 30: $59-69$.

Salganicoff, M., Sarna, M., Sax, L. and Gerstein, G.L. (1988) Unsupervised waveform classification for multi-neuron recordings: a real-time, software-based system. I. Algorithms and implementation, J. Neurosci. Methods, 25: 181-187.

Sarna, M.F., Gochin, P., Kaltenbach, J, Salganicoff, M. and Gerstein, G.L. (1988) Unsupervised waveform classification for multi-neuron recordings: a real-time, software-based system. II. Performance comparison to other sorters, J. Neurosci. Methods, 25: 189-196.

Schmidt, E.M. (1984a) Instruments for sorting neuroelectric data: a review, J. Neurosci. Methods, 12: 1-24.

Schmidt, E.M. (1984b) Computer separation of multi-unit neuroelectric data: a review, J. Neurosci. Methods, 12: 95-111.

Wörgötter, F., Daunicht, W.J, and Eckmiller, R. (1986) An on-line form discriminator for extracellular recordings based on an analog correlation technique, J. Neurosci. Methods. $17 ; 141-151$. 